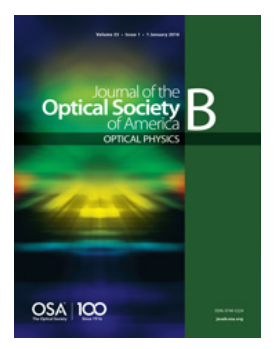

(C)2012 Optical Society of America]. One print or electronic copy may be made for personal use only. Systematic reproduction and distribution, duplication of any material in this paper for a fee or for commercial purposes, or modifications of the content of this paper are prohibited.

Available online at:

https://www . osapublishing.org/josab/abstract.cfm?uri= josab-29-12-3271

DOI: https://doi.org/10.1364/JOSAB.29.003271 


\title{
The carrier-envelope phase shift of few-cycle pulses along the focus of lenses and mirrors beyond the non-reshaping pulse approximation: the effect of pulse chirp
}

\author{
Miguel A. Porras, ${ }^{1, *}$ Balazs Major, ${ }^{2}$ and Zoltan L. Horvath ${ }^{2}$ \\ ${ }^{1}$ Departamento de Fúsica Aplicada a los Recursos Naturales, and Complex Systems Group, \\ Universidad Politécnica de Madrid, Rios Rosas 21, Madrid ES-28003, Spain \\ ${ }^{2}$ Department of Optics and Quantum Electronics, University of Szeged, P. O. Box 406, \\ H-6701 Szeged, Hungary \\ *Corresponding author: miguelangel.porras@upm.es
}

\begin{abstract}
The carrier-envelope phase shift of few-cycle pulses along a focal region is evaluated without neglecting the pulse reshaping inherent to any focusing process. The carrier-envelope phase shift is then found to strongly depend on the chirp of the focused pulse. Based on this effect, realistic optical systems for focusing few-cycle pulses are proposed to control their focal carrier-envelope phase shift for their phase-sensitive interactions with matter. (c) 2012 Optical Society of America
\end{abstract}

OCIS codes: $320.2250,320.1590,120.3620$

\section{Introduction}

A basic requirement for the observation of the sensitivity to the carrier-envelope phase (CEP) of certain light-matter interactions, such as attosecond pulse or high-harmonic generation [1-3], or for reliable measurements of the CEP of a pulse based on phase-sensitive interactions [4-6], is the constancy of the CEP in the interaction region. Significant variations of the CEP will blur the phase-sensitivity, ruining the possibility of extracting a value of the CEP. Given the high intensities usually needed, the interaction region is usually located within, or close to, the focus of a focusing system. However, it is well-known that Gouy phase causes a total CEP shift $-\pi$ during propagation through a focal region, and also that the specific CEP variation curve from the beginning to the end of the focal region depends on the focusing 
system and on the pulse in front of the focusing system. Within the approximation that the pulse temporal envelope remains unchanged during propagation through the focal region, the variation curve of the CEP has been evaluated for focusing mirrors $[7,8]$ and for lenses [9] for general pulsed Gaussian beams in front of the focusing system. In the case of focusing with lenses, the conditions under which any vestige of the dispersion introduced by the lens material can be eliminated, and under which the spherical and chromatic aberrations do not introduce any appreciable pulse broadening or distortion along the focal region, even when focusing few-cycle pulses, have been recently described in Ref. [9].

Either focused with a mirror or with a lens, and even if these optimum focusing conditions are fulfilled, the temporal envelope of few-cycle pulses may still reshape along the focal region because of their strong transversal localization. The changes on pulse form due to transversal localization, or spatiotemporal coupling effects, have been described during the last two decades in different works [10-14]; they are generally small, but more pronounced as the pulse is shorter and more transversally localized [14], and therefore are expected to be appreciable when focusing few-cycle pulses.

In this paper we evaluate the CEP evolution along the focal region of a focusing system taking into account the reshaping of the pulse during propagation through the focus due to its transversal localization, i. e., beyond the non-reshaping approximation implicit in all previous works [7-9]. We find that this reshaping does not alter previous results regarding the CEP variation curve in the focal region if the pulse is transform-limited. However, the CEP variation curve may be drastically modified if the focusing pulse is chirped, either focused with a mirror or with a lens. As an application of the dependence of focal CEP shift on pulse chirp, we show that focusing pulses with a small positive or negative chirp by a focused system with small chromatic aberration can result in a constant CEP, and therefore in an invariable temporal form of the electric field, in an important fraction of the focal region. We propose realistic focusing schemes using lenses or mirror-lens systems to produce this effect along arbitrarily long focal depths and with arbitrary focal lengths (or input spot sizes), as required in particular experimental setups.

\section{Few-cycle pulse focusing by mirrors or lenses}

We consider a focusing lens or mirror illuminated by a collimated pulsed Gaussian beam. Within the paraxial approximation, and within the Debye approximation of negligible focal shift, the temporal form of the electric field at a point in the focal region can be evaluated as the real part of the complex (analytic) field [14,15]

$$
E(t, r, z)=\frac{1}{\pi} \int_{0}^{\infty} d \omega E(\omega, r, z) \exp (-i \omega t)
$$


with the spectrum

$$
E(\omega, r, z)=p_{D}(\omega) \frac{-f}{q} \exp \left[i\left(\frac{\omega r^{2}}{2 c q}+\frac{\omega}{c} z\right)\right]
$$

where

$$
p_{D}(\omega)=p(\omega) \exp \left(i \frac{\omega}{c} D n\right) .
$$

In the above equations, $p(\omega)$ is a broad spectrum about the carrier frequency $\omega_{0}$ of the pulse specifying the amplitude and phase of each monochromatic Gaussian beam composing the input pulse. The back paraxial focal length is given by [15]

$$
\begin{aligned}
f & =\left[(n-1)\left(\frac{1}{R_{1}}+\frac{1}{R_{2}}\right)-\frac{(n-1)^{2}}{n} \frac{D}{R_{1} R_{2}}\right]^{-1} \\
& -\frac{R_{2} D}{n\left(R_{1}+R_{2}-D\right)+d}
\end{aligned}
$$

in the case of a lens with spherical surfaces of radii $R_{i}$, refraction index $n$ and center thickness $D$, or by $f=R / 2$, with $D=0$, in the case of a spherical mirror of radius $R$. The coordinate $z$ measures the distance from back vertex of the lens or mirror, $q=Z-i L_{R}$ is the complex beam parameter, $Z=z-f$ is an axial coordinate from the geometrical focus at each particular frequency $\omega, L_{R}=2 c f^{2} / \omega s^{2}$ is the half depth of focus, $s$ the input Gaussian spot size at $\omega, c$ the speed of light in vacuum, and $r$ a radial coordinate from the optical axis.

The above simple formulas from the well-known Gaussian beam propagation formalism [16] have recently been shown to provide an accurate description of the focused pulse in the case that the input spot size $s$ is much smaller than the entrance aperture radius $a_{L}$ so that the effects of truncation and of spherical aberration are negligible [9]. Under these conditions, the simple dependence of the propagated spectrum in Eqs. (2) and (3) on D implies that the dispersion introduced by the lens material is the same as that introduced by a slab of thickness equal to the lens center thickness $D$, and as such, can be easily controlled, e.g., partially or completely pre-compensated, as usually done for dielectric slabs with standard pulse shaping techniques. On the other hand, the longitudinal chromatic aberration is taken into account in Eq. (2) by the dependence of the focal length $f$ in Eq. (4) with $\omega$ in the case of the lens. Contrary to what is generally believed, chromatic aberration does not necessarily cause large pulse distortions in the focal region [9]. On the contrary, pulse broadening and distortion are negligible if the focal length variation $\sim\left|f_{0}^{\prime}\right| \Delta \omega$ within the pulse bandwidth $\Delta \omega$ is much smaller than the focal depth $2 L_{R, 0}$ (prime signs in this paper denote $d / d \omega$, and subscripts 0 evaluation at the carrier frequency $\omega_{0}$ ) [9]. In terms of the dimensionless parameter

$$
\gamma \equiv \frac{f_{0}^{\prime}}{L_{R, 0}} \omega_{0}
$$

characterizing the longitudinal chromatic aberration, this condition can be written as $|\gamma| \ll$ $\omega_{0} / \Delta \omega$ 


\section{Pulse reshaping inherent to focusing}

If the above conditions are satisfied, the temporal form of the pulse may still change because of its strong transversal localization along the focal region. It is well-known that diffraction due to transversal localization can alter the temporal form of few-cycle pulses [10-14]. Since these changes are usually small, the evolving temporal form can be suitably described by the perturbation theory for these spatiotemporal coupling effects developed in [14]. Limiting our consideration to the pulse along the optical axis $(r=0)$, we write the axial spectrum $E(\omega, 0, z)=p_{D}(\omega)(-f / q) \exp (i \omega z / c)$ as $E(\omega, 0, z)=p_{D}(\omega) a(\omega, z) \exp [i \varphi(\omega, z)]$, where

$$
a(\omega, z)=\frac{f}{L_{R}} \frac{1}{\left[1+\left(Z / L_{R}\right)^{2}\right]^{1 / 2}}
$$

and

$$
\varphi(\omega, z)=\frac{\omega}{c} z-\frac{\pi}{2}-\tan ^{-1}\left(\frac{Z}{L_{R}}\right)
$$

are the $z$-dependent parts of the spectral amplitude and phase, respectively. If the on-axis pulse is written as

$$
E(t, z)=A(t, z) \exp \left\{-i\left[\omega_{0} t-\varphi_{0}(z)\right]\right\}
$$

i.e., as enveloped carrier oscillations at the carrier frequency, and the spectral phase is approximated by $\varphi(\omega, z)=\varphi_{0}(z)+\varphi_{0}^{\prime}(z)\left(\omega-\omega_{0}\right)+\varphi_{0}^{\prime \prime}(z)\left(\omega-\omega_{0}\right)^{2} / 2$, the $z$-dependent envelope is found to be given by

$$
\begin{aligned}
A(\tau, z) & =\frac{1}{\pi} \int_{0}^{\infty} d \omega p_{D}(\omega) a(\omega, z) \\
& \times \exp \left[i \frac{1}{2} \varphi_{0}^{\prime \prime}(z)\left(\omega-\omega_{0}\right)^{2}\right] \exp \left[-i \tau\left(\omega-\omega_{0}\right)\right]
\end{aligned}
$$

where $\tau=t-\varphi_{0}^{\prime}(z)$ is the local time at position $z$, i. e., the time with origin at the instant of arrival of the pulse at $z$ in the limit of negligible pulse reshaping. The factor with $\varphi_{0}^{\prime \prime}(z)$ accounts for pulse broadening due to transversal localization, and has been seen to be negligible in all previous cases of few-cycle pulsed Gaussian beams [14]. Writing $\varphi_{0}^{\prime \prime}(z) \simeq 0$, and approaching also $a(\omega, z)=a_{0}(z)+a_{0}^{\prime}(z)\left(\omega-\omega_{0}\right)$, Eq. (9) simplifies to

$$
A(\tau, z) \simeq a_{0}(z) A_{D}(\tau)+i a_{0}^{\prime}(z) \frac{d A_{D}(\tau)}{d \tau}
$$

where

$$
A_{D}(\tau)=\frac{1}{\pi} \int_{0}^{\infty} d \omega p_{D}(\omega) \exp \left[-i \tau\left(\omega-\omega_{0}\right)\right]
$$

is the on-axis envelope at $z=0$, i.e., just after the lens or mirror. Equation (10) provides a

first-order description of the small changes of the pulse envelope during focusing. In absence of the second term in Eq. (10), the envelope would retain the same temporal form $A_{D}(\tau)$ [apart from the variable global amplitude $a_{0}(z)$ ]. The second term corrects this form to account for the transversal localization effects on few-cycle pulse shape. 


\section{The focal CEP shift}

At a point $z$ in the focal region, the CEP is the phase of the carrier oscillations at the time at which the amplitude $|A(\tau, z)|$ is maximum. If this time is $\tau_{p}$ at $z$, the CEP at $z$ is given, from Eq. (8) by $\Phi(z)=-\omega_{0}\left[\tau_{p}+\varphi_{0}^{\prime}(z)\right]+\varphi_{0}(z)+\phi$, where $\phi$ is the phase of $A\left(\tau_{p}, z\right)$. In absence of envelope reshaping, i.e., neglecting the second term in the right hand side of Eq. $(10), \tau_{p}$ and $\phi$ will be the same at any axial point $z$. The CEP shift $\Delta \Phi(z)=\Phi(z)-\Phi\left(f_{0}\right)$ from the geometrical focus $f_{0}$ at $\omega_{0}$, which is taken as the most convenient reference point, will then be given by

$$
\Delta \Phi(z)=\left[-\omega_{0} \varphi_{0}^{\prime}(z)+\varphi_{0}(z)\right]-\left[-\omega_{0} \varphi_{0}^{\prime}\left(f_{0}\right)+\varphi_{0}\left(f_{0}\right)\right] .
$$

As is well-known, this CEP shift is a consequence of the mismatch between the phase and group velocities caused by Gouy phase of the monochromatic Gaussian beam components. This is the only cause of focal CEP shift considered in all previous works [7-9], since the assumption of invariable envelope along the focal region is (more or less) implicit in all these works. Within this assumption, the most general expression found for the CEP shift from the focal point of a lens or mirror is [7-9]

$$
\Delta \Phi(z)=-\tan ^{-1} \zeta+\frac{g \zeta+\gamma \zeta^{2}}{1+\zeta^{2}},
$$

where $\zeta=Z_{0} / L_{R, 0}$ is the axial coordinate $Z_{0}=z-f_{0}$ with origin at the geometrical focus at the carrier frequency $\omega_{0}$ in units of the half focal depth $L_{R, 0}$, also at $\omega_{0}$. The parameter

$$
g=-\frac{L_{R, 0}^{\prime}}{L_{R, 0}} \omega_{0} \simeq 1+2 \frac{s_{0}^{\prime}}{s_{0}} \omega_{0}
$$

is determined by the frequency dependence about the carrier frequency of the spot size of the input monochromatic Gaussian beams. Typical (but generally unknown) values of $g$ range between 0 and 1, describing, respectively, input iso-diffracting pulsed Gaussian beams [17] (i. e., diffraction length independent of frequency) and pulsed Gaussian beam with constant spot size [10] (spot size independent of frequency). For mirrors, $\gamma=0$ since they do not introduce chromatic aberration, and for lenses, $\gamma \simeq-\left[n_{0}^{\prime} \omega_{0} /\left(n_{0}-1\right)\right]\left(f_{0} / L_{R, 0}\right)$, as obtained from Eq. (5) and the thin lens approximation of Eq. (4).

\section{The focal CEP shift beyond the non-reshaping approximation}

If however the pulse envelope reshapes with $z$, then the peak local time $\tau_{p}$ and envelope phase $\phi$ at $\tau_{p}$ will become functions of $z$. The CEP at $z$ then reads as $\Phi(z)=-\omega_{0}\left[\tau_{p}(z)+\right.$ $\left.\varphi_{0}^{\prime}(z)\right]+\varphi_{0}(z)+\phi(z)$, and its shift from $z=f_{0}$ can be written as

$$
\Delta \Phi_{\text {total }}(z)=\Delta \Phi(z)+\Delta \Phi_{C}(z)
$$


where the correction term $\Delta \Phi_{C}(z)$ is given by

$$
\Delta \Phi_{\mathrm{C}}(z)=-\omega_{0}\left[\tau_{p}(z)-\tau_{p}\left(f_{0}\right)\right]+\phi(z)-\phi\left(f_{0}\right)
$$

which is not associated to the group-phase velocity mismatch, but to the slight pulse envelope reshaping on passage through the focal region.

Let us evaluate $\Delta \Phi_{\mathrm{C}}(z)$ along a focal region when envelope reshaping is described by Eq. (10), and when the pulse immediately after the lens is the Gaussian pulse

$$
A_{D}(\tau)=\frac{\Delta t_{m}}{b} \exp \left(-\frac{\tau^{2}}{b^{2}}\right)
$$

where $b^{2}=\Delta t_{m}^{2}-2 i C, C$ is the pulse chirp, and $\Delta t_{m}$ is the Gaussian duration in absence of chirp. Under the condition $s_{0} \ll a_{L}$, the dispersion introduced by a lens reduces to that introduced by the center thickness $D$. A pulse with positive or negative linear chirp $C$ after a lens then can be obtained by over or under pre-compensating for the group delay dispersion (GDD) due to the lens center thickness. After some algebra, the derivative of the spectral amplitude in Eq. (10) with respect to frequency can be written as $a_{0}^{\prime}(z)=h(z) a_{0}(z)$, where

$$
h(z)=\frac{f_{0}^{\prime}}{f_{0}}\left(1+\frac{f_{0}}{L_{R, 0}} \frac{\zeta}{1+\zeta^{2}}\right)-\frac{L_{R, 0}^{\prime}}{L_{R, 0}}\left(1-\frac{\zeta^{2}}{1+\zeta^{2}}\right),
$$

and hence $A(\tau, z) \simeq\left[A_{D}(\tau)+i h(z)\left(d A_{D}(\tau) / d \tau\right)\right] a_{0}(z)$, which can be approached by $A(\tau, z) \simeq$ $A_{D}(\tau+i h(z)) a_{0}(z)$. Using Eq. (17) in this relation, and $1 / b^{2}=\left(1+i 2 C / \Delta t_{m}^{2}\right) / \Delta t^{2}$, where $\Delta t=\Delta t_{m}\left[1+\left(2 C / \Delta t_{m}^{2}\right)^{2}\right]^{1 / 2}$ is the duration of the chirped pulse, the amplitude at a distance $z$ after the lens is found to be

$$
|A(\tau, z)| \simeq \frac{\Delta t_{m}}{|b|} \exp \left[\frac{h^{2}(z)}{\Delta t_{m}^{2}}\right] \exp \left(-\frac{\tilde{\tau}^{2}}{\Delta t^{2}}\right) a_{0}(z),
$$

where $\tilde{\tau}=\tau-\left(2 C / \Delta t_{m}^{2}\right) h(z)$. Because of this reshaping of the amplitude, the time of maximum amplitude at $z$ is not the time $\tau_{p}=0$ expected from a simple pulse translation at the group velocity, but $\tau_{p}(z)=\left(2 C / \Delta t_{m}\right)^{2} h(z)$. According to Eq. (16), this $z$-dependent peak time results in the CEP shift $\Delta \Phi_{C}(z)=-\omega_{0}\left[\tau_{p}(z)-\tau_{p}\left(f_{0}\right)\right]=-\omega_{0}\left(2 C / \Delta t_{m}^{2}\right)\left[h(z)-h\left(f_{0}\right)\right]$, or, from Eq. (18),

$$
\Delta \Phi_{C}(z)=\frac{2 C}{\Delta t_{m}^{2}} \frac{-\gamma \zeta+g \zeta^{2}}{1+\zeta^{2}} .
$$

The variation $\phi(z)-\phi\left(f_{0}\right)$ of the phase of the envelope at $\tau_{p}(z)$ in Eq. (16) can be similarly evaluated, and is found to be irrelevant for the CEP shift. From Eqs. (13), (15) and (20), the total CEP shift from the focal point can be written as

$$
\Delta \Phi_{\text {total }}(z)=-\tan ^{-1} \zeta+\frac{G \zeta+\Gamma \zeta^{2}}{1+\zeta^{2}}
$$


i. e., in the same form as Eq. (13) in the non-reshaping approximation, but with the replacement of $g, \gamma$ with

$$
G=g-\frac{2 C}{\Delta t_{m}^{2}} \gamma, \quad \Gamma=\gamma+\frac{2 C}{\Delta t_{m}^{2}} g .
$$

Equations (21) and (22) represent our main result, which is expected to describe the actual CEP shift under the conditions explained above, namely, large aperture $\left(s_{0} \ll a_{L}\right)$ so that truncation and spherical aberration are negligible, and small chromatic aberration $(|\gamma| \ll$ $\left.\omega_{0} / \Delta \omega\right)$ so that the residual envelope reshaping is described by Eq. (10). In the Section 5 we test the validity of Eq. (21) under these conditions with a series of numerical simulations.

\section{Effect of chirp in the focal CEP shift}

We first note that for unchirped pulses $(C=0), G=g$ and $\Gamma=\gamma$. Eq. (21) then reduces to Eq. (13) obtained in the non-reshaping approximation. Thus, envelope reshaping, if any, does not involve any additional CEP shift for unchirped focused pulses, as obtained, e.g., by complete pre-compensation for the dispersion due to the lens center thickness. Let us briefly recall the cases of particular interest and main results in the non-reshaping approximation, since these results hold beyond this approximation for unchirped pulses. The CEP variation curve about the focus has a horizontal inflection point at $z=f_{0}$ for $g=1$ [Fig. 1(a, dotted curve)], i. e., for an input pulse with spot size independent of frequency [10,14]. However, most experiments $[6,7]$ suggest $g \sim 0$, i. e., approximately input isodiffracting Gaussian beam [17], since the observed CEP variation along the focal region of a mirror $(\gamma=0)$ matches approximately the Gouy phase shift, as predicted by Eq. (13) with $g=0$ [Fig. 1(a, dashed curve)]. Replacing the mirror with a lens with chromatic aberration such that $\gamma=-1$, the CEP becomes nearly constant before the focus [9] [Fig. 1(a, solid curve)]. From the expression $\gamma \simeq-\left[n_{0}^{\prime} \omega_{0} /\left(n_{0}-1\right)\right]\left(f_{0} / L_{R, 0}\right)$ for a lens, and from $L_{R, 0}=2 c f_{0}^{2} / \omega_{0} s_{0}^{2}$, the input spot size and focal length

$$
s_{0}=\sqrt{\frac{2 c L_{R, 0}}{\omega_{0}}} \frac{n_{0}-1}{n_{0}^{\prime} \omega_{0}}, \quad f_{0}=\sqrt{\frac{\omega_{0} L_{R, 0}}{2 c}} s_{0},
$$

must be used to obtain this effect in a given half-depth of focus $L_{R, 0}[9]$. We note, however, that the CEP continues to have the same pronounced variation at $z=f_{0}$ as with the mirror, since the slope of the CEP curve at focus is only determined by $g$, i.e., by the variation of the spot size of the input pulse with $\omega$.

Equations (21) and (22) predict that if envelope reshaping is taken into account the above results are not valid for chirped pulses, since the CEP shift becomes dependent on pulse chirp. According to Eqs. (21) and (22) the most relevant effect of chirp is to change the slope of the CEP curve at focus of a lens $(\gamma<0)$. This can qualitatively be understood from the sketch in Fig. 1(b). For $C>0$, bluer spectral components (dashed blue curves) are placed 

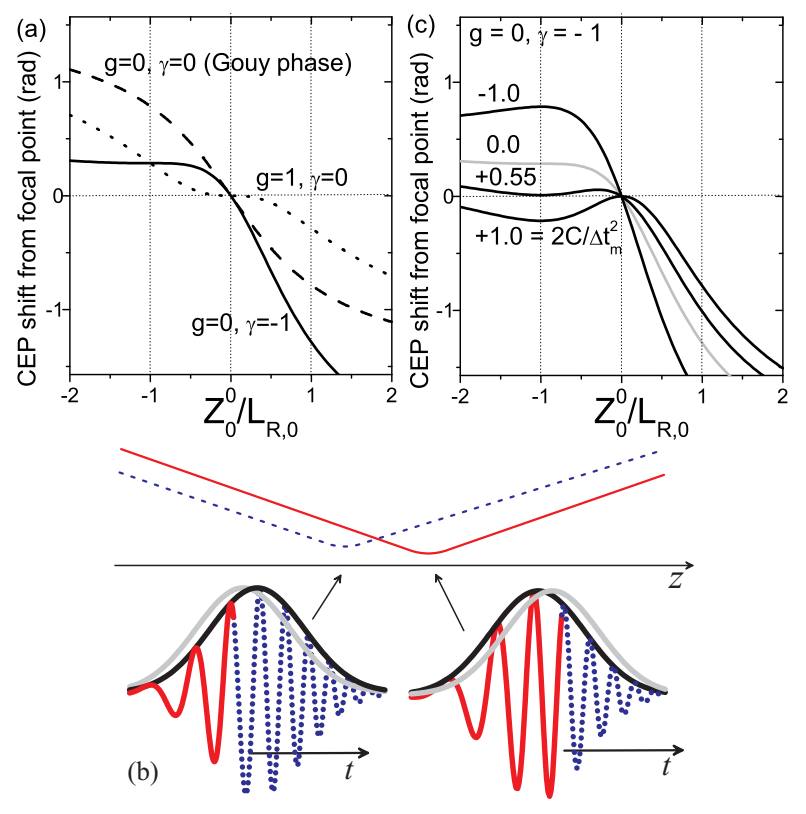

Fig. 1. (a) CEP shifts from the focal point predicted by Eq. (13) in the nonreshaping approximation for pulsed Gaussian beams with spot size independent of frequency focused by a mirror $(g=1, \gamma=0)$ (dotted curve), isodiffracting pulsed Gaussian beams focused by a mirror $(g=0, \gamma=0)$ (dashed curve), and isodiffracting pulsed Gaussian beams focused by a lens $(g=0, \gamma=-1)$. (b) (Color online) Sketch of the effect of chirp on envelope reshaping when focusing with small chromatic aberration. Bluer spectral components (dashed blue curves) placed in the trailing part of the pulse are focused at shorter distances, and redder spectral components (solid red curves) in the leading part, at larger distances. (c) CEP shifts from the focal point predicted by Eq. (21) for isodiffracting pulsed Gaussian beams $(g=0, \gamma=-1)$ with increasing chirps. 
in the trailing part of the pulse, and are focused by a lens at $z<f_{0}$, becoming relatively enhanced in amplitude in this region. At $z>f_{0}$ the same is true for redder components (solid red curves). The pulse peak of this reshaping envelope (black Gaussian curves) then traverses the focal region faster than expected from its group velocity (gray Gaussian curves), lowering its mismatch from the superluminal phase velocity due to Gouy phase. In the case that $C<0$ the mismatch increases. CEP curves of focused pulses with negative, zero and positive chirps are shown in Fig. 1(c) in the case of focusing with the optimum spot size and lens given by Eq. (23) $(g=0, \gamma=-1)$. As an application, we note that the small positive chirp

$$
\frac{2 C}{\Delta t_{m}^{2}} \simeq+0.55
$$

involving the small pulse broadening $\Delta t \simeq 1.1 \Delta t_{m}$, causes the CEP slope at focus to diminish so that the region of nearly constant $\mathrm{CEP}$ extends to reach the focus. For instance, the $\mathrm{CEP}$ shift in $\left[-L_{R, 0}, 0\right]$ diminishes from $16^{\circ}$ to $4^{\circ}$ when the pulse is chirped with $2 C / \Delta t_{m}^{2} \simeq+0.55$.

\section{Example and numerical verification}

As a verification, we have simulated numerically the focusing of pulses at $800 \mathrm{~nm}$ carrier wave length $\left(\omega_{0}=2.355 \mathrm{fs}^{-1}\right)$ of transform-limited duration $\Delta t_{m}=4.53 \mathrm{fs}$ (FWHM duration 5.34 fs, or two-cycle pulses) with a realistic fused silica lens to a half focal depth $L_{R, 0}=2.5 \mathrm{~mm}$. With a fused silica lens, focusing to constant CEP before the focus $(\gamma=-1)$ requires, from Eqs. (23), the input spot size $s_{0}=0.827 \mathrm{~mm}$ for the input iso-diffracting pulsed Gaussian beam $\left[s=s_{0}\left(\omega_{0} / \omega\right)^{1 / 2}\right.$ for $\left.g=0\right]$, and the focal length $f_{0}=81.96 \mathrm{~mm}$. We choose the lens to have front and back surface radii $R_{1}=R_{2}=74.46 \mathrm{~mm}$, center thickness $D=1 \mathrm{~mm}$, to give, from Eq. (4) the required focal length $f_{0}$ at the carrier frequency, and lens aperture radius to be $a_{L}=5 \mathrm{~mm} \gg s_{0}$. The pulse shapes about the focus and their CEPs are evaluated with the numerical method described in detail in [9]. In this hybrid ray-tracing/wave-propagation spectral method, Snell's law through the spherical surfaces of the lens is used to obtain the output wave front just behind the lens and its effective aperture for each frequency. The output wave fronts for each frequency are propagated up to the focal region using Fresnel diffraction in the Debye approximation [15], and the temporal pulse forms along the optical axis $(r=0)$ are evaluated from the inverse Fourier transform in Eq. (1). The effects of lens material dispersion through the variable thickness of the lens, the effect of the lens aperture truncation, and of spherical and chromatic aberrations, are then accurately taken into account in this procedure.

The input spectrum is taken to be $p(\omega)=\exp \left[-\Delta t_{m}^{2}\left(\omega-\omega_{0}\right)^{2} / 4\right] \exp \left[-i\left(\varphi_{0}^{\prime \prime}-C\right)(\omega-\right.$ $\left.\left.\omega_{0}\right)^{2} / 2-i \varphi_{0}^{\prime \prime \prime}\left(\omega-\omega_{0}\right)^{3} / 6\right]$, where the third-order dispersion $\varphi_{0}^{\prime \prime \prime}=32.11 \mathrm{fs}^{3}$ is introduced in order to pre-compensate for that due to the lens center thickness $D$, and the GDD $\varphi_{0}^{\prime \prime}-C=$ $36.75 \mathrm{fs}^{2}-C$ is introduced in order to under or over pre-compensate for that due to the lens 


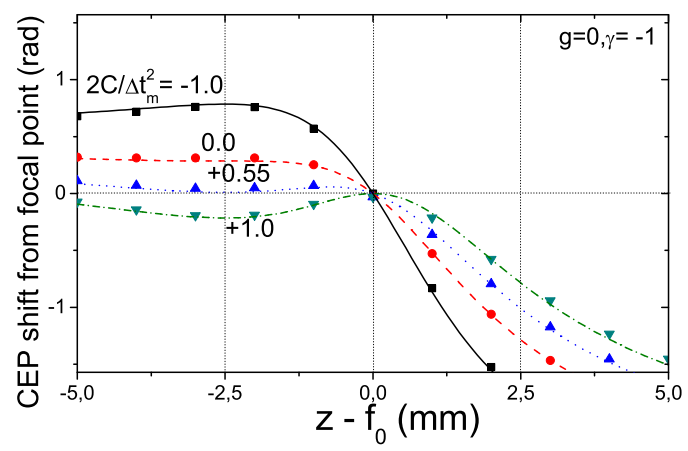

Fig. 2. (Color online) For focused Gaussian pulses with increasing chirps, CEP shifts from the focal point along the focal region of a fused silica lens, evaluated numerically (symbols) and predicted by Eq. (21) (curves). See text for details about the input pulse, the lens characteristics, and the numerical method.
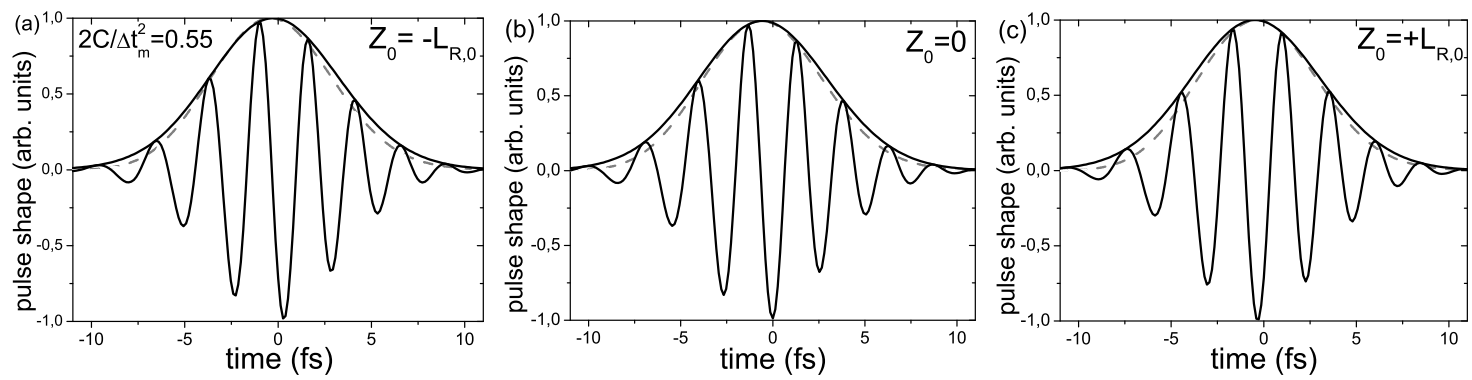

Fig. 3. Axial pulse shapes and envelopes (a) at the beginning, (b) at the middle and (c) at the end of the focal region for the input pulse and fused silica lens described in the text. The pulse chirps are $2 C / \Delta t_{m}^{2}=0.55$ (solid curves) and $2 C / \Delta t_{m}^{2}=0$ (dashed curves). 
center thickness, obtaining in this way an approximately Gaussian pulse with chirp $C$ after the lens.

Figure 2 shows that the CEP shifts, extracted from the numerically evaluated pulse temporal forms, for focused pulses with the different chirps $2 C / \Delta t_{m}^{2}=-1,0,+0.55,+1$ (different symbols) fit accurately to those predicted by our approximate analytical formula in Eq. (21) (solid, dashed, dotted and dash-dotted curves). In the particular case of $2 C / \Delta t_{m}^{2}=+0.55$, the numerically evaluated pulse shapes and envelopes at the positions $Z_{0}=-L_{R, 0}, 0,+L_{R, 0}$ are represented by the solid curves in Figs. 3(a), (b) and (c). For comparison, the corresponding envelopes in absence of chirp are represented by dashed curves in the same figure. In absence of chirp, envelope reshaping due to transversal localization is negligible. The pulse remains a nearly Gaussian pulse of the transform-limited duration $\Delta t_{m}=4.53$ fs along the entire focal region because the conditions $s_{0}=0.827 \ll a_{L}=5 \mathrm{~mm}$ and $|\gamma|=1 \ll \omega_{0} / \Delta \omega \simeq 4.5$ are satisfied. The chirp $2 C / \Delta t_{m}^{2}=+0.55$ needed to obtain the flattest CEP curve is so small that it is barely noticeable in the oscillations of the electric field, the focused pulse being just slightly broadened through the entire focal region in comparison to the unchirped pulse [Figs 3(a), (b) and (c)]. The small, but still perceptible, envelope reshaping caused by the transversal localization substantially results in a slight temporal shift of the envelope, which is retarded in front of the focus $\left(Z_{0}=-L_{R, 0}\right)$, and advanced behind the focus $\left(Z_{0}=+L_{R, 0}\right)$, as in the qualitative sketch in Fig. 1(b). Since reshaping is mainly a temporal shift of the envelope that causes the CEP variation to be negligible in the first half of the focal region, the temporal shape of the electric field remains unaltered from $Z_{0}=-L_{R, 0}$ to $Z_{0}=0$, as it can be seen in Figs. 3(a) and (b).

\section{Further applications}

The use of small chirps and chromatic aberrations allow to tailor other focal CEP variation curves for specific applications. As seen in Fig. 4, the CEP can also be frozen in the second half of the focal region, where the gas jet in high-order harmonic generation experiments is usually placed, by negatively chirping the pulse with $2 C / \Delta t_{m}^{2}=-0.55$ and focusing with a system with small positive chromatic aberration such that $\gamma=+1$. The simplest example of such a system is a mirror with short focal length $f_{M}$ close enough to a diverging lens of large focal length $f_{L}<0$ so that the total focal length is given by $1 / f=1 / f_{M}+1 / f_{L}$ and remains positive. For this system, Eq. (5) yields

$$
\gamma \simeq-\frac{f_{0}^{2}}{f_{L, 0}^{2}} \frac{n_{0}^{\prime} \omega_{0}}{n_{0}-1} \frac{f_{L, 0}}{L_{R, 0}}
$$

An advantageous feature of this system is that the spot size $s_{0}$ in front of the system to get a frozen the CEP is not fixed by the desired depth of focus $L_{R, 0}$, as in Eq. (23) in the case of a single lens, but both $s_{0}$ and $L_{R, 0}$ can be chosen freely according to experimental 


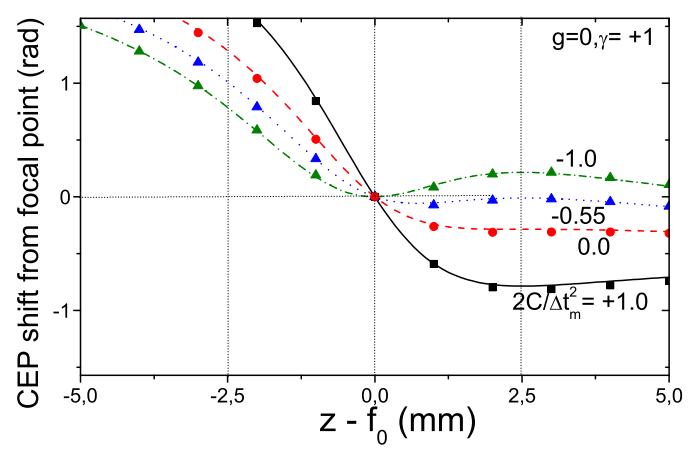

Fig. 4. (Color online) For focused Gaussian pulses with different chirps, CEP shifts along the focal region of the mirror-lens system described in the text, evaluated numerically (symbols) and from Eq. (12) (curves). See the text for details about the input pulse and focusing system.

needs. For example, expanded input beams with spot sizes of the order of one centimeter are frequently used in experiments, this requirement being particularly relevant to avoid heating and nonlinear effects if a lens is to be used. Chosen $s_{0}$ and $L_{R, 0}$ according to the experimental requirements, the condition $\gamma=+1$ in Eq. (25) and the relation $L_{R, 0}=2 c f_{0}^{2} / \omega_{0} s_{0}^{2}$ lead to the lens focal length

$$
\frac{1}{f_{L, 0}}=-\frac{n_{0}-1}{n_{0}^{\prime} \omega_{0}} \frac{2 c}{\omega_{0} s_{0}^{2}}
$$

to the mirror focal length $1 / f_{M}=1 / f_{0}-1 / f_{L, 0}$, with the total focal length $f_{0}=$ $\left(\omega_{0} L_{R, 0} / 2 c\right)^{1 / 2} s_{0}$. The negative chirp $2 C / \Delta t_{m}^{2} \simeq-0.55$ in the focusing pulse may result from over pre-compensation of the dispersion due to the center thickness of the diverging lens. For example, focusing a pulse at $800 \mathrm{~nm}$ with the large input spot size $s_{0}=10 \mathrm{~mm}$ to freeze the CEP in $L_{R, 0}=2.5 \mathrm{~mm}$ requires the system of focal length $f_{0}=991 \mathrm{~mm}$, formed, e.g., by a mirror with $f_{M}=877 \mathrm{~mm}$ and a $\mathrm{CaF}_{2}$ lens with $f_{L, 0}=-7632 \mathrm{~mm}$. Similar values are obtained if a fused silica lens, or other typical lens materials are used. In a realistic simulation using the same method as outlined above, we have chosen the radius of the spherical mirror $2 f_{M}$, a $\mathrm{CaF}_{2}$ lens with $R_{1}=2 f_{M}, R_{2}=-1143.5 \mathrm{~mm}$, center thickness $D=0.2 \mathrm{~mm}$ and aperture $a_{L}=40 \mathrm{~mm} \gg s_{0}$. The input pulses of transform-limited Gaussian duration $\Delta t_{m}=4.53 \mathrm{fs}$ contain, as above, pre-compensation for the lens third-order dispersion and partial pre-compensation for the GDD to obtain the values of $2 C / \Delta t_{m}^{2}$ indicated in Fig. 4. As seen in this Figure, the numerically evaluated CEP values (symbols) fit again to the predictions of the analytical formula in Eq. (21) (curves), which supports the practicability of this method of CEP control with these scalable focusing systems. 


\section{Conclusions}

We have evaluated the focal CEP shift of few-cycle pulses along the focal region of focusing systems beyond the usual approximation that pulse reshaping along the focal region is negligible. We considered the effect of the primary, or intrinsic pulse reshaping due to transversal localization, which is the only origin of reshaping along the focal region under the conditions of negligible spherical and chromatic aberrations with lenses or mirrors. The CEP shift along the focal region, and therefore the change of the temporal form of the electric field, is then found to be influenced by the chirp of the focused pulse, and is accurately described by Eqs. (21) and (22). Based on this result, small pulse chirp and chromatic aberration can be used to further stabilize the CEP of the focused pulse against the CEP shift imposed by Gouy phase in a large fraction of the focal region. Simple focusing systems, as a single lens or a mirror-lens system, can be designed to produce this effect along any needed half-depth of focus for input pulses with arbitrary spot size (e. g., in the centimeter range) at the usual carrier wave lengths in phase-sensitive light-matter interaction experiments, or equivalently, with arbitrary system focal lengths (e.g., of the order of one meter).

\section{References}

1. F. Krausz and Misha Yu. Ivanov, "Attosecond Physics," Rev. Mod. Phys. 81, 161-234 (2009).

2. C. A. Haworth, L. E. Chipperfield, J. S. Robinson, P. L. Knight, J. P. Marangos and J. W. G. Tisch, "Half-cycle cutoffs in harmonic spectra and robust carrier-envelope phase retrieval," Nature Physics 3, 52-57 (2007).

3. M. Schnüurer, Z. Cheng, M. Hentschel, F.Krausz, T.Wilhein, D.Hambach, G. Schmahl, M. Drescher, Y. Lim, U. Heinzmann, "Few-cycle-driven XUV laser harmonics: generation and focusing," Appl. Phys. B 70, S227-S232 (2000).

4. T. Wittmann, B. Horvath, W. Helml, M. G. Schtzel, X. Gu, A. L. Cavalieri, G. G. Paulus, and R. Kienberger, "Single-shot carrier-envelope phase measurement of few-cycle laser pulses," Nature Physics 5, 357-362 (2009).

5. G.G. Paulus, F. Lindner, H.Walther, A. Baltuska, E. Goulielmakis, M. Lezius, and F. Krausz, "Measurement of the Phase of Few-Cycle Laser Pulses," Phys. Rev. Lett. 91, 253004 (2003).

6. F. Lindner, G.G. Paulus, H.Walther, A. Baltuska, E. Goulielmakis, M. Lezius, and F. Krausz, "Gouy Phase Shift for Few-Cycle Laser Pulses," Phys. Rev. Lett. 92, 113001 (2004).

7. T. Tritschler, K. D. Hof, M. W. Klein, and M. Wegener, "Variation of the carrier-envelope phase of few-cycle laser pulses owing to the Gouy phase: a solid-state-based measurement," Opt. Lett. 30, 753-755 (2005). 
8. M. A. Porras, "Characterization of the electric field of focused pulsed Gaussian beams for phase-sensitive interactions with matter," Opt. Lett. 34, 1546-1548 (2009).

9. M. A. Porras, Z. L. Horvath, and B. Major, "On the use of lenses to focus few-cycle pulses with controlled carrier-envelope phase," Applied Physics B: Lasers and Optics 108, 521-531 (2012).

10. I. P. Christov, "Propagation of femtosecond light pulses," Opt. Commun. 53, 364-366 (1985).

11. C.J.R. Sheppard and X. Gan, "Free-space propagation of femto-second light pulses," Opt. Commun. 133, 1-6 (1997)

12. A. E. Kaplan, "Diffraction-induced transformation of near-cycle and sub-cycle pulses," J. Opt. Soc. Am. B 15, 951-956 (1998).

13. G. P. Agrawal, "Spectrum-induced changes in diffraction of pulsed optical beams," Opt. Commun. 157, 52-56 (1998)

14. M. A. Porras, "Diffraction effects in few-cycle optical pulses," Phys. Rev. E 65, 026606 (2002).

15. M. Born and E. Wolf, Principles of Optics, Pergamon Press, Oxford (1987).

16. See, for example, A. E. Siegman, Lasers, University Science books, Mill Valley, California (1986).

17. M. A. Porras, "Ultrashort pulsed Gaussian beams," Phys. Rev. E 58, 1086-1093 (1998). 